(n)

trobertivier

Journal of Nonlinear Mathematical Physics

ISSN (Online): 1776-0852 ISSN (Print): 1402-9251

$f^{\text {rinumsis }}$ Journal Home Page: https://www.atlantis-press.com/journals/jnmp

Symmetric waves are traveling waves for a shallow water equation modeling surface waves of moderate amplitude

Anna Geyer

To cite this article: Anna Geyer (2015) Symmetric waves are traveling waves for a shallow water equation modeling surface waves of moderate amplitude, Journal of Nonlinear Mathematical Physics 22:4, 545-551, DOI:

https://doi.org/10.1080/14029251.2015.1129492

To link to this article: https://doi.org/10.1080/14029251.2015.1129492

Published online: 04 January 2021 


\title{
Symmetric waves are traveling waves for a shallow water equation modeling surface waves of moderate amplitude
}

\author{
Anna Geyer \\ Departament de Matemàtiques, Facultat de Ciències, \\ Universitat Autònoma de Barcelona, 08193 Bellaterra, Spain. \\ annageyer@mat.uab.cat
}

Received 27 September 2015

Accepted 12 October 2015

\begin{abstract}
Following a general principle introduced by Ehrnström, Holden and Raynaud in 2009, we prove that for an equation modeling the free surface evolution of moderate amplitude waves in shallow water, all symmetric waves are traveling waves.
\end{abstract}

Keywords: symmetric waves; traveling waves; free surface; shallow water; moderate amplitude.

2010 Mathematics Subject Classification: 35Q35; 35B06; 35D30;

\section{Introduction}

In this note we are concerned with the relation between symmetric and traveling wave solutions of a model equation for surface waves of moderate amplitude in shallow water

$$
u_{t}+u_{x}+6 u u_{x}-6 u^{2} u_{x}+12 u^{3} u_{x}+u_{x x x}-u_{x x t}+14 u u_{x x x}+28 u_{x} u_{x x}=0,
$$

which arises as an approximation of the Euler equations in the context of homogenous, inviscid gravity water waves propagating over a flat bed. The equation was originally derived by Johnson [20] by means of formal asymptotic expansions from the Euler equations. These considerations were followed up by Constantin and Lannes [6] who proved that the equation approximates the governing equations to the same order as the Camassa-Holm equation [2], which models the horizontal fluid velocity at a certain depth beneath the fluid [20] (see also [19] for an alternative derivation of the $\mathrm{CH}$ equation by a variational approach). An important feature of Eq. (1.1) is that it captures the non-linear phenomenon of wave breaking $[6,10]$ in the sense that the surface profile remains bounded but its slope may form singularities in finite time, see the general discussion in $[4,31]$. The Cauchy problem associated to (1.1) is locally well-posed in $H^{s}$, for $s>3 / 2$, on the real line as well as on the circle $[11,13]$, and the data-to-solution map was shown to be non-uniformly continuous [13]. For a well-posedness result in the context of Besov spaces we refer the reader to [27], whereas a result on low regularity solutions with $1<s \leq 3 / 2$ may be found in [24], and for global conservative solutions and continuation of solutions beyond wave breaking we point out [32]. The equation admits smooth as well as cusped and peaked solitary and periodic traveling wave solutions, and also solitary traveling waves with compact support [15-17]. Furthermore, the smooth solitary traveling wave solutions are known to be orbitally stable [12].

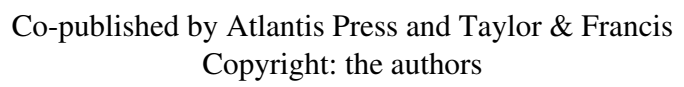


In the present note we are concerned with symmetry properties of solutions of (1.1). A vast amount of research has been conducted into considerations regarding symmetry in the context of water waves, for model equations as well for the full governing equations. It is astonishing that all steady water waves known to exist are symmetric and that in many cases symmetry can be established a priori, see for example $[8,14,21]$ for solitary and periodic waves in irrotational flows, $[3,5,7]$ for periodic water waves with vorticity, [30] for stratified water waves, and [18] for equatorial wind waves. A number of characterizations of symmetric waves in various settings also point towards the importance of this defining feature in the analysis of water waves, see for instance [25] for rotational solitary waves, [29] for periodic water waves with stagnation points, and [26] for a characterization of symmetric steady water waves in terms of the underlying flow.

Following a general principle introduced by Ehrnström et.al. in [9], our main result states that a symmetric wave solution of Eq. (1.1) is necessarily a traveling wave. Conversely, we know from the analysis carried out in $[15,17]$ that all solitary and periodic traveling wave solutions (smooth as well as peaked or cusped) are symmetric about their crest and trough. In light of these considerations we establish the identity between symmetric and solitary and periodic traveling wave solutions of (1.1), cf. Remark 2.1. We point out that similar results hold for the Camassa-Holm equation [9] and for the Whitham equation [1].

\section{Main Result}

Following [9] and the general principle deduced therein, we make the following definition for symmetric waves.

Definition 2.1. A solution $u$ is $x$-symmetric if there exists a function $\lambda \in \mathscr{C}^{1}\left(\mathbb{R}_{+}\right)$such that for every $t>0$,

$$
u(t, x)=u(t, 2 \lambda(t)-x)
$$

for a.e. $x \in \mathbb{R}$. We say that $\lambda(t)$ is the axis of symmetry.

The equation for surface waves (1.1) fulfills the formal requirements of the general principle stated in Theorem 2.2 of [9] whose proof is carried out for classical solutions. It is possible, however, to modify the argument to extend the solution space beyond differentiable functions and to accommodate for weak solutions. To this end, we write the Eq. (1.1) in weak non-local form as

$$
u_{t}-\partial_{x}\left(u+7 u^{2}\right)+\partial_{x}\left(1-\partial_{x}^{2}\right)^{-1} R(u)=0, \quad x \in \mathbb{R}, t>0,
$$

where

$$
R(u):=2 u+10 u^{2}-2 u^{3}+3 u^{4}-7 u_{x}^{2} .
$$

Note that this equation holds in $H^{1}(\mathbb{R})$. Indeed, let $P(x):=\left(1-\partial_{x}^{2}\right)^{-1} R(u)=\frac{1}{2} e^{-|x|} * R(u)$, then $P \in H^{1}(\mathbb{R})$ for $u \in H^{1}(\mathbb{R})$ by Young's inequality. We define weak solutions of Eq. (1.1) in the following way.

Definition 2.2. A function $u(t, x)$ is a weak solution of Eq. (1.1) if $u \in \mathscr{C}\left(\mathbb{R}_{+}, H^{1}(\mathbb{R})\right)$ satisfies

$$
\iint_{\mathbb{R}_{+} \times \mathbb{R}} u \phi_{t}-\left(u+7 u^{2}\right) \phi_{x}+\left(1-\partial_{x}^{2}\right)^{-1} R(u) \phi_{x} d t d x=0,
$$

for all $\phi \in \mathscr{C}_{0}^{\infty}\left(\mathbb{R}_{+} \times \mathbb{R}\right)$. 
We are now ready to state our main result.

Theorem 2.1. Let $u$ be a weak solution of (1.1) with initial data such that the equation is locally well-posed. If $u$ is $x$-symmetric then $u$ is a traveling wave solution.

Proof. We follow [9] and introduce the notation

$$
f_{\lambda}(t, x):=f(t, 2 \lambda(t)-x)
$$

Let $\phi \in \mathscr{C}_{0}^{1}\left(\mathbb{R}_{+}, \mathscr{C}_{0}^{3}\right)$ be a test function and note that the definition 2.2 for weak solutions of (1.1) remains valid, since $\mathscr{C}_{0}^{\infty}\left(\mathbb{R}_{+} \times \mathbb{R}\right)$ is dense in $\mathscr{C}_{0}^{1}\left(\mathbb{R}_{+}, \mathscr{C}_{0}^{3}\right)$. Furthermore, $\mathscr{C}_{0}^{1}\left(\mathbb{R}_{+}, \mathscr{C}_{0}^{3}\right)$ is invariant under $\phi \rightarrow \phi_{\lambda}$ because $\lambda \in \mathscr{C}^{1}$, and in view of $\left(\phi_{\lambda}\right)_{\lambda}=\phi$ we find that this transformation is bijective. Let $u$ be an $x$-symmetric solution of (1.1), then, using bracket notation for distributions, $u$ satisfies

$$
<u, \phi_{t}>-<u+7 u^{2}, \phi_{x}>+<\left(1-\partial_{x}^{2}\right)^{-1} R(u), \phi_{x}>=0 .
$$

Since $u$ is symmetric, we have $u=u_{\lambda}$ and we find that $\left\langle u_{\lambda}, \phi>=-<u, \phi_{\lambda}\right\rangle$, and $\left\langle u_{\lambda}^{2}, \phi>=\right.$ $-<u^{2}, \phi_{\lambda}>$, and

$$
\begin{aligned}
<\left(1-\partial_{x}^{2}\right)^{-1} R\left(u_{\lambda}\right), \phi> & =\iint_{\mathbb{R}_{+} \times \mathbb{R}} \frac{1}{2} e^{-|x|} * R(u(t, 2 \lambda-x)) \phi(t, x) d t d x \\
& =\iint_{\mathbb{R}_{+} \times \mathbb{R}} \int_{\mathbb{R}} \frac{1}{2} e^{-|s|} R(u(t, 2 \lambda-(x-s))) d s \phi(t, x) d t d x \\
& =-\iint_{\mathbb{R}_{+} \times \mathbb{R}} \int_{\mathbb{R}} \frac{1}{2} e^{-|s|} R(u(t, x+s)) d s \phi(t, 2 \lambda-x) d t d x \\
& =-\iint_{\mathbb{R}_{+} \times \mathbb{R}} \frac{1}{2} e^{-|x|} * R(u(t, x)) \phi(t, 2 \lambda-x) d t d x \\
& =-<\left(1-\partial_{x}^{2}\right)^{-1} R(u), \phi_{\lambda}>.
\end{aligned}
$$

Furthermore, we have that

$$
\left(\phi_{\lambda}\right)_{t}=\left(\phi_{t}\right)_{\lambda}-2 \dot{\lambda}(t)\left(\phi_{x}\right)_{\lambda}, \quad\left(\phi_{\lambda}\right)_{x}=-\left(\phi_{x}\right)_{\lambda} .
$$

Since $u_{\lambda}$ satisfies (2.5) we have in view of the above identities that

$$
\begin{aligned}
0 & =<u,\left(\phi_{t}\right)_{\lambda}>-<u+7 u^{2},\left(\phi_{x}\right)_{\lambda}>+<\left(1-\partial_{x}^{2}\right)^{-1} R(u),\left(\phi_{x}\right)_{\lambda}> \\
& =<u,\left(\phi_{\lambda}\right)_{t}-2 \dot{\lambda}(t)\left(\phi_{\lambda}\right)_{x}>+<u+7 u^{2},\left(\phi_{\lambda}\right)_{x}>-<\left(1-\partial_{x}^{2}\right)^{-1} R(u),\left(\phi_{\lambda}\right)_{x}>.
\end{aligned}
$$

Taking $\phi=\phi_{\lambda}$ in the above equation, we get

$$
<u, \phi_{t}-2 \dot{\lambda}(t) \phi_{x}>+<u+7 u^{2}, \phi_{x}>-<\left(1-\partial_{x}^{2}\right)^{-1} R(u), \phi_{x}>=0,
$$

since $\left(\phi_{\lambda}\right)_{\lambda}=\phi$. We subtract the latter equation from (2.5) and obtain

$$
<u, \dot{\lambda}(t) \phi_{x}>-<u+7 u^{2}, \phi_{x}>+<\left(1-\partial_{x}^{2}\right)^{-1} R(u), \phi_{x}>=0 .
$$

Consider now the sequence

$$
\phi_{\varepsilon}(t, x)=\psi(x) \rho_{\varepsilon}(t)
$$


where $\rho_{\varepsilon} \in \mathscr{C}_{0}^{\infty}(\mathbb{R})$ is a mollifier with the property that $\rho_{\varepsilon}(t) \rightarrow \delta\left(t-t_{0}\right)$, the Dirac delta function with mass in $t_{0}$, as $\varepsilon \rightarrow 0$. Using this sequence in Eq. (2.6) we obtain

$$
\int_{\mathbb{R}} \psi_{x}(x)\left[\int_{\mathbb{R}_{+}} \dot{\lambda}(t) u(t, x) \rho_{\varepsilon}(t)-\left(u+7 u^{2}\right)(t, x) \rho_{\varepsilon}(t)+\left(1-\partial_{x}^{2}\right)^{-1} R(u(t, x)) \rho_{\varepsilon}(t) d t\right] d x=0 .
$$

By assumption we have that $u \in \mathscr{C}\left(\mathbb{R}_{+}, H^{1}(\mathbb{R})\right)$, and therefore

$$
\lim _{\varepsilon \rightarrow 0} \int_{\mathbb{R}_{+}} u(t, x) \rho_{\varepsilon}(t) d t=u\left(t_{0}, x\right)
$$

in $L^{2}(\mathbb{R})$ and

$$
\lim _{\varepsilon \rightarrow 0} \int_{\mathbb{R}_{+}} u^{2}(t, x) \rho_{\varepsilon}(t) d t=u^{2}\left(t_{0}, x\right)
$$

in $L^{1}(\mathbb{R})$. Furthermore, we have that

$$
\lim _{\varepsilon \rightarrow 0} \int_{\mathbb{R}_{+}}\left(1-\partial_{x}^{2}\right)^{-1} R(u(t, x)) \rho_{\varepsilon}(t) d t=\left(1-\partial_{x}^{2}\right)^{-1} R\left(u\left(t_{0}, x\right)\right)
$$

in $L^{2}(\mathbb{R})$, since $\left(1-\partial_{x}^{2}\right)^{-1} R(u) \in H^{1}(\mathbb{R}) \subset L^{2}(\mathbb{R})$, cf. [28]. Therefore, in the limit when $\varepsilon$ tends to zero, (2.7) implies that $u\left(t_{0}, x\right)$ satisfies

$$
\int_{\mathbb{R}}\left(-\dot{\lambda}\left(t_{0}\right) u+u+7 u^{2}\right) \psi_{x}-\left(1-\partial_{x}^{2}\right)^{-1} R(u) \psi_{x} d x=0,
$$

for all $\psi \in \mathscr{C}_{0}^{\infty}(\mathbb{R})$. Set now $c:=-\dot{\lambda}\left(t_{0}\right)$ and define $\bar{u}(t, x)=u\left(t_{0}, x+c\left(t-t_{0}\right)\right)$. We claim that the function $\bar{u}$ is a weak traveling wave solution of (1.1). If this is true the result follows immediately, since then $\bar{u}\left(t_{0}, x\right)=u\left(t_{0}, x\right)$ and in view of uniqueness of solutions we find that $\bar{u}(t, x)=u(t, x)$ for all $t$. Therefore, $u$ is a traveling wave solution of (1.1) as we wanted to show. To prove the claim, we demonstrate that if a function $U \in H^{1}(\mathbb{R})$ satisfies

$$
\int_{\mathbb{R}}\left(c U+U+7 U^{2}\right) \psi_{x}-\left(1-\partial_{x}^{2}\right)^{-1} R(U) \psi_{x} d x=0
$$

for all $\psi \in \mathscr{C}_{0}^{\infty}(\mathbb{R})$, then the function

$$
\bar{u}(t, x)=U\left(x-c\left(t-t_{0}\right)\right)
$$

is a weak solution of (1.1). To see that this is true, notice that $\bar{u}$ defined as above belongs to $\mathscr{C}\left(\mathbb{R}, H^{1}(\mathbb{R})\right)$ since the translation map is continuous from $\mathbb{R}$ into $H^{1}(\mathbb{R})$ and $t \rightarrow c\left(t-t_{0}\right)$ is real analytic. We may assume that $t_{0}=0$ and for any function $\phi \in \mathscr{C}_{0}^{\infty}\left(\mathbb{R}_{+} \times \mathbb{R}\right)$ use the shorthand

$$
\phi_{c}(t, x):=\phi(t, x+c t) .
$$


Then we have that $<\bar{u}, \phi>=<U, \phi_{c}>$ and

$$
\begin{aligned}
<\left(1-\partial_{x}^{2}\right)^{-1} R(\bar{u}), \phi> & =\iint_{\mathbb{R}_{+} \times \mathbb{R}} \frac{1}{2} e^{-|x|} * R(U(x-c t)) \phi(t, x) d t d x \\
& =\iint_{\mathbb{R}_{+} \times \mathbb{R}} \int_{\mathbb{R}} \frac{1}{2} e^{-|s|} R(U(x-c t-s)) d s \phi(t, x) d t d x \\
& =\iint_{\mathbb{R}_{+} \times \mathbb{R}} \int_{\mathbb{R}} \frac{1}{2} e^{-|s|} R(U(r-s)) d s \phi(t, r+c t) d t d r \\
& =\iint_{\mathbb{R}_{+} \times \mathbb{R}} \frac{1}{2} e^{-|r|} * R(U(r)) \phi(t, r+c t) d t d r \\
& =<\left(1-\partial_{x}^{2}\right)^{-1} R(U), \phi_{c}>.
\end{aligned}
$$

In view of

$$
\left(\phi_{c}\right)_{t}=\left(\phi_{t}\right)_{c}+c\left(\phi_{x}\right)_{c}, \quad\left(\phi_{x}\right)_{c}=\left(\phi_{c}\right)_{x}
$$

we obtain

$$
\begin{aligned}
& <\bar{u}, \phi_{t}>-<\bar{u}+7 \bar{u}^{2}, \phi_{x}>+<\left(1-\partial_{x}^{2}\right)^{-1} R(\bar{u}), \phi_{x}> \\
& =<U,\left(\phi_{c}\right)_{t}-c\left(\phi_{c}\right)_{x}>-<U+7 U^{2},\left(\phi_{c}\right)_{x}>+<\left(1-\partial_{x}^{2}\right)^{-1} R(U),\left(\phi_{c}\right)_{x}>.
\end{aligned}
$$

Observe that, since $U$ is independent of time, we have

$$
\begin{aligned}
<U,\left(\phi_{c}\right)_{t}> & =\iint_{\mathbb{R}_{+} \times \mathbb{R}} U(x-c t) \partial_{t} \phi_{c}(t, x) d t d x \\
& =\int_{\mathbb{R}_{+}} U(s)\left(\int_{\mathbb{R}_{t}} \partial_{t} \phi_{c}(t, s+c t) d t\right) d s \\
& =\int_{\mathbb{R}_{+}} U(s)\left(\phi_{c}(T, s+c T)-\phi_{c}(0, s)\right) d s=0,
\end{aligned}
$$

where $T$ is large enough to be outside of the support of $\phi_{c}$. Therefore,

$$
\begin{aligned}
& <\bar{u}, \phi_{t}>-<\bar{u}+7 \bar{u}^{2}, \phi_{x}>+<\left(1-\partial_{x}^{2}\right)^{-1} R(\bar{u}), \phi_{x}> \\
& =-c<U,\left(\phi_{c}\right)_{x}>-<U+7 U^{2},\left(\phi_{c}\right)_{x}>+<\left(1-\partial_{x}^{2}\right)^{-1} R(U),\left(\phi_{c}\right)_{x}> \\
& =\iint_{\mathbb{R}_{+} \times \mathbb{R}}-\left(c U+U+7 U^{2}\right)\left(\phi_{c}\right)_{x}+\left(1-\partial_{x}^{2}\right)^{-1} R(U)\left(\phi_{c}\right)_{x} d t d x=0,
\end{aligned}
$$

since $U \in H^{1}$ satisfies (2.9) with $\psi(x)=\phi_{c}(t, x)$ which belongs to $\mathscr{C}_{0}^{\infty}(\mathbb{R})$ for every given $t \geq 0$. This shows that $\bar{u}$ is a weak solution of (1.1) which proves the claim and concludes the proof.

Remark 2.1. Theorem 2.1 states that all $x$-symmetric solutions of Eq. (1.1) are traveling wave solutions. Conversely, we know from [15] and [17] that all smooth, peaked and cusped solitary traveling waves are symmetric with respect to their crests or troughs, and likewise for periodic traveling waves on each period as long as they conserve the energy of the associated planar system. Indeed, for smooth solutions this follows immediately from the fact that the planar system corresponding to the ordinary differential equation satisfied by the traveling wave solutions of (1.1) has a first integral which is symmetric in its second variable, and smooth traveling wave solutions of (1.1) correspond to smooth homoclinic or periodic orbits of the planar differential system which are symmetric with 
respect to the horizontal axis and defined on the entire real line. On the other hand, orbits corresponding to solutions of (1.1) whose first derivative is not continuous or blows up in a countable number of points are heteroclinic or homoclinic connections whose alpha or omega limits tend to or reach a line of singularities in the phase plane. The corresponding solutions are therefore defined only on a subset of $\mathbb{R}$ and may be continued to the whole line by joining these wave segments, cf. [15, 17]. In this way, it is possible to obtain a plethora of weak solutions with complex shapes, as demonstrated by Lenells [22,23] for the Camassa-Holm and Degasperis-Procesi, which are in general clearly not symmetric. However, if one requires that this continuation of solutions conserves the energy of the planar system, i.e. if one imposes that the orbit remains in the same level set of the first integral, then the solution composed of wave segments in this way is necessarily symmetric. The same is true for the Camassa-Holm equation: the weak traveling wave solutions which are not symmetric are the ones composed of wave segments corresponding to orbits with different energy.

\section{Acknowledgements}

The author is supported by the FWF project J 3452 "Dynamical Systems Methods in Hydrodynamics" of the Austrian Science Fund. Part of this work was carried out during a research visit at NTNU Trondheim for which the author gratefully acknowledges support.

\section{References}

[1] G. Brüll, M. Ehrnström and L. Pei, Symmetry and decay properties of travelling wave solutions to the Whitham equation, preprint

[2] R. Camassa and D. D. Holm, An integrable shallow water equation with peaked solitons, Phys. Rev. Lett. 71(11) (1993) 1661-1664.

[3] A. Constantin, M. Ehrnström and E. Wahlén, Symmetry of steady periodic gravity water waves with vorticity, Duke Math. J. 140(3) (2007) 591-603.

[4] A. Constantin and J. Escher, Wave breaking for nonlinear nonlocal shallow water equations, Acta Math. 181(2) (1998) 229-243.

[5] A. Constantin and J. Escher, Symmetry of steady periodic surface water waves with vorticity, J. Fluid Mech. 498 (2004) 171-181.

[6] A. Constantin and D. Lannes, The hydrodynamical relevance of the Camassa-Holm and DegasperisProcesi equations, Arch. Ration. Mech. Anal. 192 (2009) 165-186.

[7] A. Constantin and W. Strauss, Exact steady periodic water waves with vorticity., Comm. Pure Appl. Math. 57(4) (2004) 481-527.

[8] W. Craig and P. Sternberg, Symmetry of solitary waves, Comm. Partial Differ. Equations 13(5) (1988) 603-633.

[9] M. Ehrnström, H. Holden and X. Raynaud, Symmetric Waves Are Traveling Waves, Int. Math. Res. Not. 2009(24) (2009) 4578-4596.

[10] N. Duruk Mutlubaş, On the Cauchy problem for a model equation for shallow water waves of moderate amplitude, Nonlinear Anal. Real World Appl. 14(5) (2013) 2022-2026.

[11] N. Duruk Mutlubaş, Local well-posedness and wave breaking results for periodic solutions of a shallow water equation for waves of moderate amplitude, Nonlinear Anal. Theory, Methods Appl. 97 (2014) $145-154$.

[12] N. Duruk Mutlubaş and A. Geyer, Orbital stability of solitary waves of moderate amplitude in shallow water, J. Differ. Equations 255(2) (2013) 254-263.

[13] N. Duruk Mutlubaş, A. Geyer and B.-V. Matioc, Non-uniform continuity of the flow map for an evolution equation modeling shallow water waves of moderate amplitude, Nonlinear Anal. Real World Appl. 17 (2014) 322-331.

[14] P. Garabedian, Surface waves of finite depth, J. d'Analyse Math. 14(1) (1965) 161-169. 
[15] A. Gasull and A. Geyer, Traveling surface waves of moderate amplitude in shallow water, Nonlinear Anal. Theory, Methods Appl. 102 (2014) 105-119.

[16] A. Geyer, Solitary traveling water waves of moderate amplitude, J. Nonl. Math. Phys. 19(Suppl. 1) (2012) p. 1240010.

[17] A. Geyer and V. Mañosa, Singular solutions for a class of traveling wave equations arising in hydrodynamics, submitted .

[18] D. Henry and A.-V. Matioc, On the existence of equatorial wind waves, Nonlinear Anal. Theory, Methods Appl. 101 (2014) 113-123.

[19] D. Ionescu-Kruse, Variational derivation of the Camassa-Holm shallow water equation, J. Nonlinear Math. Phys. 14(3) (2007) 311-320.

[20] R. S. Johnson, Camassa-Holm, Korteweg-de Vries and related models for water waves, J. Fluid Mech. 455 (2002) 63-82.

[21] F. Kogelbauer, Symmetric irrotational water waves are traveling waves, J. Differ. Equ. 259(10) (2015) 5271-5275.

[22] J. Lenells, Traveling wave solutions of the Camassa-Holm equation, J. Differ. Equ. 217(2) (2005) 393430.

[23] J. Lenells, Traveling wave solutions of the Degasperis-Procesi equation, J. Math. Anal. Appl. 306(1) (2005) 72-82.

[24] X. Liu and J. Liu, On the low regularity solutions and wave breaking for an equation modeling shallow water waves of moderate amplitude, Nonlinear Anal. Theory, Methods Appl. 107 (2014) 1-11.

[25] A.-V. Matioc and B.-V. Matioc, Regularity and symmetry properties of rotational solitary water waves, J. Evol. Equations 12(2) (2012) 481-494.

[26] B.-V. Matioc, A characterization of the symmetric steady water waves in terms of the underlying flow, Discret. Contin. Dyn. Syst. Ser. A 34(8) (2014) 3125-3133.

[27] Y. Mi and C. Mu, On the solutions of a model equation for shallow water waves of moderate amplitude, J. Differ. Equations 255(8) (2013) 2101-2129.

[28] W. Rudin, Principles of Mathematical Analysis, 3rd edn. (McGraw-Hill Book Company, Inc., New York-Toronto-London, 1976).

[29] G. Tulzer, On the symmetry of steady periodic water waves with stagnation points, Commun. Pure Appl. Anal. 11(4) (2012) 1577-1586.

[30] S. Walsh, Some criteria for the symmetry of stratified water waves, Wave Motion 46 (2009) 350-362.

[31] G. B. Whitham, Linear and Nonlinear Waves (Wiley \& Sons, New York, 1980).

[32] S. Zhou and C. Mu, Global conservative solutions for a model equation for shallow water waves of moderate amplitude, J. Differ. Equ. 256(5) (2014) 1793-1816. 